\title{
Estudio de la actividad antifúngica de materiales conteniendo carbón proveniente de pilas en desuso
}

\author{
Study of the antifungal activity of materials \\ containing coal from disused batteries
}

Katerine Igal ${ }^{1}$, Natalia Bellotti ${ }^{2}$, Jorge Sambeth ${ }^{1}$, Patricia Vázquez ${ }^{1}$

\author{
${ }^{1}$ Centro de Investigación y Desarrollo en Ciencias Aplicadas "Dr. Jorge J. Ronco"- CINDECA - CCT CONICET La \\ Plata/Universidad Nacional de La Plata CP: 1900, La Plata, Buenos Aires, Argentina. \\ e-mail: katerineigal@gmail.com, sambeth@quimica.unlp.edu.ar,vazquez@quimica.unlp.edu.ar \\ ${ }^{2}$ Centro de Investigación y Desarrollo en Tecnología de Pinturas -CIDEPINT -CIC-CONICET Calle 52 e/ 121 y 122, CP: \\ 1900La Plata, Buenos Aires, Argentina. \\ e-mail: pinturashigienicas@cidepint.gov.ar
}

\section{RESUMEN}

La gran cantidad de pilas que se descartan y el impacto ambiental que la inadecuada disposición de los residuos de las mismas produce impulsó a su reciclado. Tanto las pilas alcalinas como las de cinc-carbón tienen como componentes principales cinc y carbón mezclado con otros óxidos, en su relleno interno.

En este trabajo se realizó la síntesis de sílice a través del método sol-gel a partir del precursor tetraetilortosilicato y del catalizador hidróxido de amonio, incorporando carbón proveniente del reciclado de las pilas en desuso en $0,1,1,0$ y $10,0 \mathrm{C} \%(\mathrm{p} / \mathrm{p})$, respectivamente. Este soporte fue funcionalizado con el 3aminopropiltrietoxisilano, con una relación 1:1 y, posteriormente, fue impregnado mediante la técnica de humedad incipiente con nitrato de plata, dando como resultado 1 y 2 Ag \% (p/v) adsorbido. Estos sólidos se caracterizaron mediante las técnicas de $\mathrm{S}_{\mathrm{BET}}$, DRX, titulación potenciométrica, SEM-EDX y TEM. Se evaluó la actividad antifúngica de los sólidos obtenidos frente a los hongos Chaetomium globosum, Aspergillus sp. y Cladosporium sp., mediante el test de inhibición en placa. Los mejores resultados obtenidos mostraron un grado de inhibición cercano al $100 \%$ frente a Cladosporium sp. con ambas concentraciones de Ag. En una siguiente etapa se realizarán modificaciones en la síntesis del material silíceo para aumentar su efectividad como antifúngico.

Palabras clave: sílice, carbón, nitrato de plata, antimicrobiano.

\section{ABSTRACT}

The large number of batteries that are discarded and the environmental impact that the inadequate disposition of the waste of the same produces impelled to its recycling. Both alkaline and zinc-carbon batteries have as their main components zinc and carbon mixed with other oxides, in their internal fill.

In this work, the silica synthesis was carried out using the sol-gel method from the tetraethylorthosilicate precursor and the ammonium hydroxide catalyst, carbon from the recycling of disused batteries was added in 0.1 , 1.0 and $10.0 \mathrm{C} \%(\mathrm{w} / \mathrm{w})$, respectively. This support was functionalized by 3-aminopropyltriethoxysilane in a 1: 1 ratio and subsequently it was impregnated by the incipient wetness with silver nitrate, resulting in 1 and $2 \% \mathrm{Ag}(\mathrm{w} / \mathrm{v})$ adsorbed. These solids were characterized by $\mathrm{S}_{\mathrm{BET}}$, DRX, potentiometric titration, SEM-EDX and TEM techniques. The antifungal activity of the solids was assessed against the fungi Chaetomium globosum, Aspergillus sp. and Cladosporium sp. by plaque inhibition test. The best results obtained showed a degree of inhibition close to $100 \%$ against Cladosporium sp. with samples impregnated at both Ag concentrations. In a next stage modifications will be made in the synthesis of the siliceous material to increase its antifungal effectiveness.

Keywords: silica, carbon, silver nitrate, antimicrobial.

\section{INTRODUCCIÓN}


Las consecuencias nocivas producidas sobre nuestro planeta debido al desarrollo tecnológico son innegables, y muchas veces, irreversibles. Dentro de los factores que impactan negativamente en el medioambiente se encuentran el uso indiscriminado de pilas que se descartan en forma masiva mediante una inadecuada disposición de las mismas. De acuerdo a los Principios de la Química Verde que tienen como fin minimizar la contaminación y desarrollar nuevos métodos menos agresivos con el medio ambiente se impulsó al reciclado de las pilas en desuso [1]. Tanto las pilas alcalinas como las de cinc-carbón tienen como componentes principales: cinc y carbón, mezclado con otros óxidos, en su relleno interno [2].

Los microorganismos en condiciones de humedad y temperatura adecuadas crecen formando biopelículas sobre distintos materiales, entre ellos, las telas. El tejido y otros materiales textiles, especialmente los hechos de fibras naturales, tales como algodón y lana, pueden actuar como medio para el crecimiento de microorganismos tales como bacterias y hongos asociados con infecciones y alergias entre otras afecciones. Por lo tanto, hay un creciente interés por la salud e higiene personal, los textiles con propiedades antimicrobianas se están convirtiendo en un objetivo cada vez más deseable para los fabricantes de estos materiales [3]. Los agentes antimicrobianos son sustancias químicas sintéticas o de origen natural que impiden la colonización microbiológica [4]. Es sabido que los compuestos y los iones de plata son altamente tóxicos para los microorganismos que muestran fuerte efecto biocida en muchas especies de bacterias [5]. Según datos bibliográficos de estudios realizados con bacterias las nanopartículas de plata ejercer una actividad bactericida predominantemente a través de la liberación de iones plata seguido por una mayor permeabilidad de la membrana, fuga del contenido celular y disrupción de la replicación del ADN. La estabilidad de las nanopartículas de plata se rige por su tamaño y forma y, también, influye el pH de la solución, la fuerza iónica, los iones específicos y los ligandos en su biodisponibilidad [6]. Para estabilizar la estructura de las nanopartículas, controlar la concentración de las mismas o iones metálicos liberados, prolongar el tiempo de liberación y, por lo tanto, mejorar la durabilidad y la resistencia al lavado en las telas, los metales nanosificados y los óxidos metálicos se han incluido en matrices poliméricas, tales como matrices siliceas [5, 7, 8].

El objetivo de este trabajo plantea la obtención de matrices silíceas modificadas con carbón provenientes del reciclado de pilas para su uso como material antifúngico. El primer paso fue realizar la síntesis de sílice a través del método sol-gel, a partir del precursor tetraetilortosilicato y del catalizador hidróxido de amonio, incorporando el carbón en los siguiente porcentajes: 0,1, 1,0 y 10,0 C \% (p/p), respectivamente. Este soporte fue funcionalizado con el 3-aminopropiltrietoxisilano, con una relación 1:1 [9]. Posteriormente fue impregnado mediante la técnica de humedad incipiente con nitrato de plata, dando como resultado 1 y $2 \mathrm{Ag}$ $\%(\mathrm{p} / \mathrm{v})$ adsorbido [10].

\section{MATERIALES Y MÉTODOS}

\subsection{Síntesis de las muestras a partir del método sol-gel}

Se utilizó el método sol-gel en atmósfera de nitrógeno para sintetizar los sólidos basados en sílice. El tetraetilortosilicato $\left(\mathrm{Si}\left(\mathrm{OC}_{2} \mathrm{H}_{5}\right)_{4}, 98 \%\right)$ (TEOS), se utilizó como precursor de la sílice y el hidróxido de amonio $\left(\mathrm{NH}_{4} \mathrm{OH}, 28 \%\right)$ como catalizador, mientras que en todas las muestras sintetizadas el etanol absoluto $\left(\mathrm{CH}_{2} \mathrm{CH}_{3} \mathrm{OH}, 99,9 \%\right)(\mathrm{EtOH})$ y el agua destilada fueron usados como disolventes. A los sólidos se les incorporó carbón, proveniente del reciclado de las pilas en desuso, en 0,1, 1,0 y 10,0\% (p/p). Las muestras se dejaron secar a temperatura ambiente, hasta tener peso constante, y luego se calcinaron a $150{ }^{\circ} \mathrm{C}$ durante $2 \mathrm{~h}$. La nomenclatura que se usara será para la sílice pura: $\mathrm{KB}$; para el carbón reciclado: $\mathrm{C}$ y para la sílice con agregados de C, en forma creciente al porcentaje será: K1B; K2B y K3B, respectivamente.

Estos soportes fueron funcionalizados mediante un compuesto, el 3-aminopropiltrietoxisilano (APS) con una relación 1:1. Denominándose: K1Bf; K2Bf y K3Bf y, posteriormente, fueron impregnados mediante la técnica de humedad incipiente con nitrato de plata dando como resultado 1 y $2 \mathrm{Ag} \%$ (p/v) adsorbido. Se dejaron durante 1 semana a temperatura ambiente para su secado. Estos se denominaran de acuerdo a la cantidad de Ag, para 1\%: K1BfAg; K2BfAg y K3BfAg y para 2\%: K1Bf2Ag; K2Bf2Ag y K3Bf2Ag, respectivamente.

\subsection{Caracterización de las muestras sintetizadas}

Se realizó la caracterización de las muestras mediante las siguientes técnicas: titulación potenciométrica con $n$-butilamina, adsorción-desorción de $\mathrm{N}_{2}\left(\mathrm{~S}_{\mathrm{BET}}\right)$, difracción de rayos $\mathrm{X}(\mathrm{DRX})$, microscopía electrónica de barrido (SEM), imágenes digitales LG G Pro Lite 8MP y microscopía electrónica de transmisión (TEM). La estimación de las propiedades ácidas de los materiales se realizó por medio de titulación potenciométrica con n-butilamina. Se llevó a cabo en un equipo Metrohm 794 Basic Titrino con un electrodo de doble unión. Se 
agregaron $0,025 \mathrm{ml} / \mathrm{min}$ de solución de $n$-butilamina en acetonitrilo $(0,05 \mathrm{~N})$ a una cantidad conocida $(0,05$ g) del sólido de interés, previamente suspendido en acetonitrilo $(90 \mathrm{ml})$, y agitado por un período de $3 \mathrm{~h}$. Las propiedades texturales, como el área superficial de los sólidos $\left(\mathrm{S}_{\mathrm{BET}}\right)$, se determinaron mediante un equipo Micromeritics Accusorb 2100, utilizan-do $\mathrm{N}_{2}$ como gas adsorbible. Los diagramas de rayos X (DRX) se realizaron con un equipo Philips modelo PW-1390 (control de canal) y PW-1394 (control de motor), con registrador gráfico de barrido incorporado. Se usó radiación $\mathrm{Cu} \mathrm{K \alpha}(\alpha=1,5417 \AA)$, filtro de níquel, $20 \mathrm{~mA}$ y $40 \mathrm{kV}$ en la fuente de alta tensión, ángulo de barrido (20) comprendido entre $5^{\circ}$ y $60^{\circ}$, velocidad de barrido de $2 \%$ min y amplitud de la escala vertical 2000 cuentas/seg. Se realizó microscopía electrónica de barrido (SEM) para la obtención de micrografías de los sólidos, empleandoun equipo Philips Modelo 505, a un potencial de trabajo de $15 \mathrm{kV}$, soportando las muestras sobre grafito y metalizándolas con oro. El equipo utilizado para TEM fue um microscopio electrónico de transmisión JEOL, modelo $100 \mathrm{CX}$, operado a $100 \mathrm{kV}$. Las muestras se prepararon suspendiendo el material en etanol y llevándolo 15 min a baño ultrasónico para favorecer la dispersión. Posteriormente, se colocaron gotas de esta suspensión sobre una grilla provista con un film de Formvar® y se dejó secar 30 min. Todo el procedimiento se realizó a temperatura ambiente.

\subsection{Actividad antifúngica}

La evaluación de la actividad antifúngica de los sólidos se realizó mediante el ensayo de inhibición en placa frente a Chaetomium globosum, Aspergillus sp. y Cladosporium sp. Se realizaron subcultivos de los aislamientos mencionados en placas de Petri. La composición del medio de cultivo agarizado utilizado (MCA) fue: $1,5 \mathrm{~g}$ agar, $1 \mathrm{~g}$ dextrosa, $0,5 \mathrm{~g}$ proteasa peptona, $0,1 \mathrm{~g} \mathrm{KH}_{2} \mathrm{PO}_{4}, 0,05 \mathrm{~g} \mathrm{MgSO}{ }_{4} .7 \mathrm{H}_{2} \mathrm{O}$ y agua destilada. Se prepararon placas con $15 \mathrm{ml}$ de medio de cultivo y con las muestras en una concentración de 60 ppm y 120 ppm en relación al contenido de Ag, respectivamente. Las placas fueron inoculadas con $20 \mu \mathrm{l}$ de la suspensión de esporas e incubadas a $28^{\circ} \mathrm{C}$. Luego de 10 días se registró el crecimiento, en cada caso, se obtuvo el diámetro promedio a partir de tres medidas independientes. El ensayo se realizó por triplicado.

\section{RESULTADOS Y DISCUSIÓN}

La titulación potenciométrica con $n$-butilamina permite determinar las propiedades ácidas de una dispersión de partículas sólidas, a través de la medida de la diferencia de potencial del electrodo (E). Asimismo, para interpretar los resultados, se sabe que el potencial de electrodo inicial (Ei) indica la máxima fuerza ácida de los sitios superficiales y los valores (meq/g sólido), donde se alcanza el plateau, indica el número total de sitios ácidos. La fuerza ácida de los sitios superficiales puede clasificarse de acuerdo a los siguientes rangos: sitio muy fuerte, Ei $>100 \mathrm{mV}$, sitio fuerte, $0<\mathrm{Ei}<100 \mathrm{mV}$; sitio débil, $-100<\mathrm{Ei}<0 \mathrm{mV}$, y sitio muy débil, $\mathrm{Ei}<-100 \mathrm{mV}$. En la Tabla 1 se presentan los valores de acidez inicial de las muestras (Ei) con el agregado de $\mathrm{C}$ y en la Fig. 1 pueden observarse las curvas potenciométricas obtenidas para las muestras: C, KB, K1B, $\mathrm{K} 2 \mathrm{~B}, \mathrm{~K} 3 \mathrm{~B}$ y K3Bf.

Tabla 1: Composición química y propiedades acidas de las muestras sintetizadas.

\begin{tabular}{l|l|l}
\hline Muestras & $\begin{array}{l}{[\mathrm{C}]} \\
(\% \mathrm{p} / \mathrm{p})\end{array}$ & $\begin{array}{l}\mathrm{Ei} \\
(\mathrm{mV})\end{array}$ \\
\hline KB & 0 & 83,1 \\
\hline $\mathrm{C}$ & 100 & 37,1 \\
\hline K1B & 0,1 & 112 \\
\hline K2B & 1,0 & 91,8 \\
\hline K3B & 10,0 & 82,4 \\
\hline K3Bf & 10,0 & $-13,9$ \\
\hline
\end{tabular}


Puede observarse que la sílice pura, obtenida mediante hidrolisis básica, presenta un Ei inicial de 83,1 $\mathrm{mV}$ mientras que el $\mathrm{C}$ adquirido de las pilas recicladas tiene un valor cercano a los $40 \mathrm{mV}$, lo que presenta en la comparación de los sitios ácidos que ambos se encuentran en la zona de sitios débiles. Si se observan los valores obtenidos con el agregado de carbón en la síntesis de la sílice estos varían entre 112 y 82,4 mV, manteniéndose más cercanos a los valores de la sílice pura que a los del C, como era esperable por las pequeñas cantidades que se adicionaron. Estos cambios son visibles en la Fig. 1A donde las curvas continúan un comportamiento semejante al de la sílice no así a la curva de C. En la Fig. 1B se comparan las curvas de la muestra con contenido de carbón más alto y la misma muestra una vez funcionalizada con APS. Aquí la diferencia es notoria ya que la funcionalización elimina los sitios con escaza acidez que pudieran quedar en la superficie de la sílice, dando una curva por debajo de $0 \mathrm{mV}$.

A

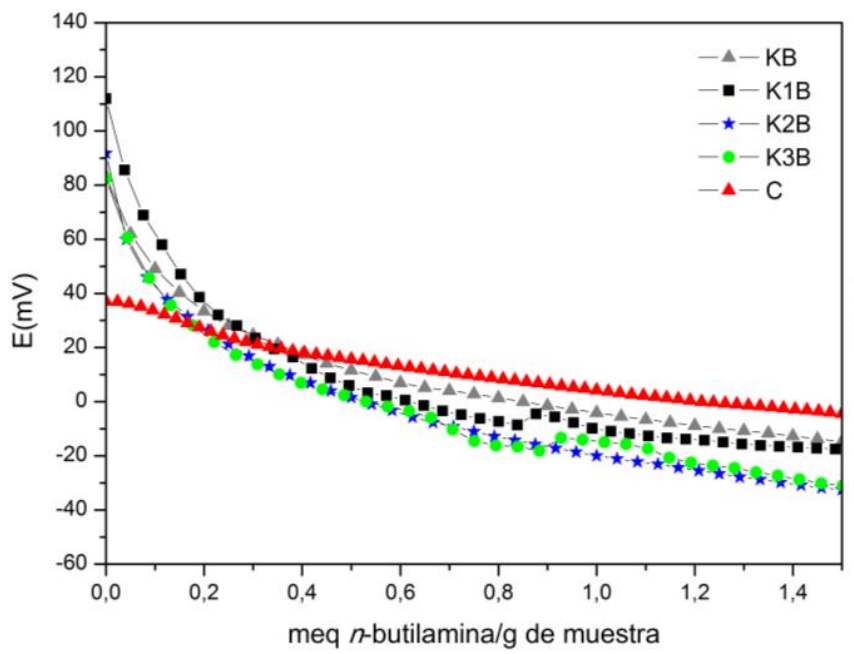

B

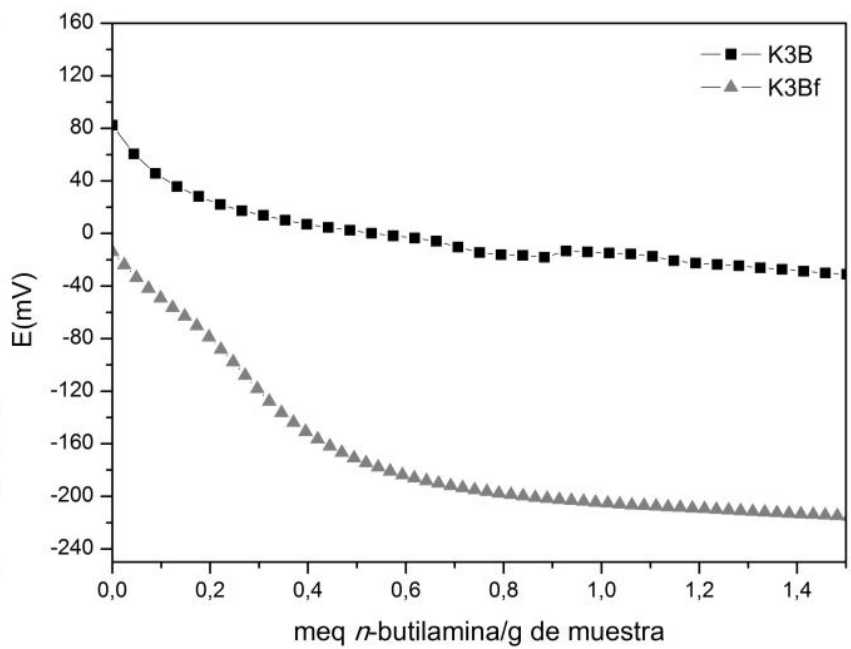

Figura 1A y 1B : Curvas potenciometricas de las muestras sintetizadas.

Siguiendo con la caracterización de los materiales, una manera de determinar el área superficial, tamaño y volumen de poros en materiales porosos es utilizando la adsorción de nitrógeno. Cualquier átomo o molécula que se aproxime a una superficie experimenta un potencial neto atractivo debido a la deficiencia de enlaces que presenta la superficie con respecto al bulk. Las propiedades texturales de las muestras sintetizadas se presentan en la Tabla 2. Para cada muestra se presenta $S_{\mathrm{BET}}$, en $\mathrm{m}^{2} / \mathrm{g}$, el volumen de poros, en $\mathrm{cm}^{3} / \mathrm{g}$, y el tamaño medio de poros, en $\AA$, además de los valores obtenidos por el Método t-plot de Boer.

Las muestras obtenidas con hidróxido de amonio presentaron valores muy bajos de superficie específica, encontrándose en el error de la técnica, con excepción de la K1B. Por otra parte, los valores de volumen de poros correspondientes a estas muestras son casi nulos, dando total imprecisión a las otras medidas, por ejemplo, tamaño de poros.

Tabla 2: Propiedades estructurales de las muestras sintetizadas con contenido de C.

\begin{tabular}{c|l|c|c|c}
\hline Muestras & $\begin{array}{l}\mathrm{S}_{\mathrm{BET}} \\
\left(\mathrm{m}^{2} / \mathrm{g}\right)\end{array}$ & $\begin{array}{c}\text { Volumen de } \\
\text { Poro }\left(\mathrm{cm}^{3} / \mathrm{g}\right)\end{array}$ & $\begin{array}{c}\text { Diámetro de } \\
\text { Poro }(\AA)\end{array}$ & T- plot \\
\hline KB & 5,8 & 0,01 & 68,8 & 2,6 \\
\hline K1B & 12,4 & 0,01 & 49,2 & 1,3 \\
\hline K2B & 3,5 & 0,005 & 64,7 & 2,1 \\
\hline K3B & 7,4 & 0,01 & 81,1 & 1,3 \\
\hline
\end{tabular}

En la Figura 2 se presentan las curvas de adsorción-desorción de $\mathrm{N}_{2}$ de las muestras sintetizadas con TEOS como precursor, mostrándose la sílice KB1 y la KB3, respectivamente, ya que las restantes muestras dieron curvas similares. La forma de las isotermas puede dar información sobre el tipo de porosidad del sólido. Las isotermas correspondientes a muestras obtenidas usando hidróxido de amonio como catalizador po- 
drían incluirse en las de tipo II, características de sólidos poco porosos, con meso y macroporosidad. El punto $\mathrm{B}$ es donde el cubrimiento de la monocapa es completo y está por empezar la adsorción en multicapa. Esta clase de isoterma es característica de sólidos no-porosos o de adsorbentes macroporosos.
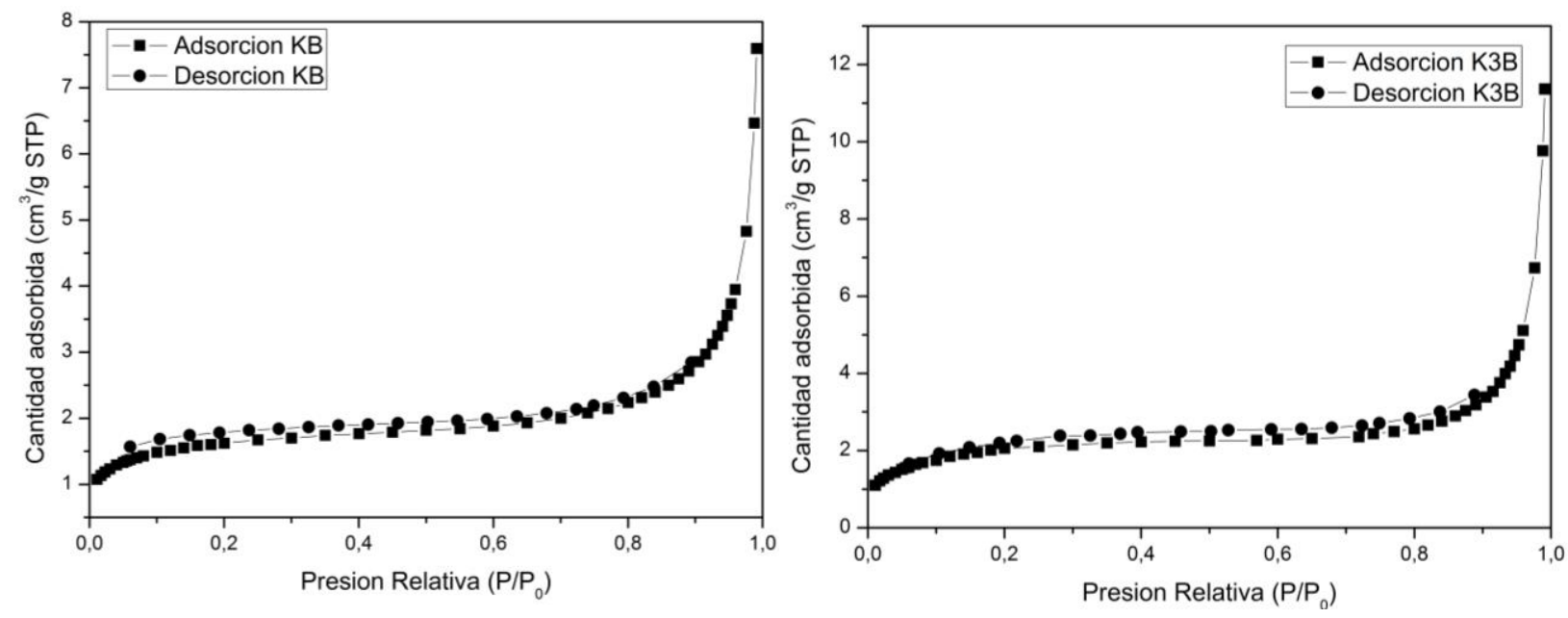

Figura 2: Isotermas de adsorción-desorción de $\mathrm{N}_{2}$ de las muestras KB y K3B.

En el presente trabajo la determinación de la estructura de los materiales sintetizados se llevó a cabo mediante la técnica de difracción de rayos X (XRD). En la Figura 3, a modo de ejemplo, se presentan los diagramas de difracción de rayos $\mathrm{X}$ de las muestras de KB, KB3, KB3f y KB3fAg, respectivamente. Se confirmó el carácter amorfo de los xerogeles de sílice hidrófilos que presentan solamente amplios picos en el intervalo $15-30^{\circ} 2 \theta$ y se observó la banda ubicada alrededor de $23^{\circ} 2 \theta$, que es la difracción típica de este tipo de sílice. La comparacion de los diagramas de KB y KB3 muestran una señal que podria deberse la impurezas del carbon; esta señal disminuye su tamaño al ser funcionalizada puediendo deberse a la oclusion de los mismos por el APS, tornandose pequeña cuando se impregna con Ag.

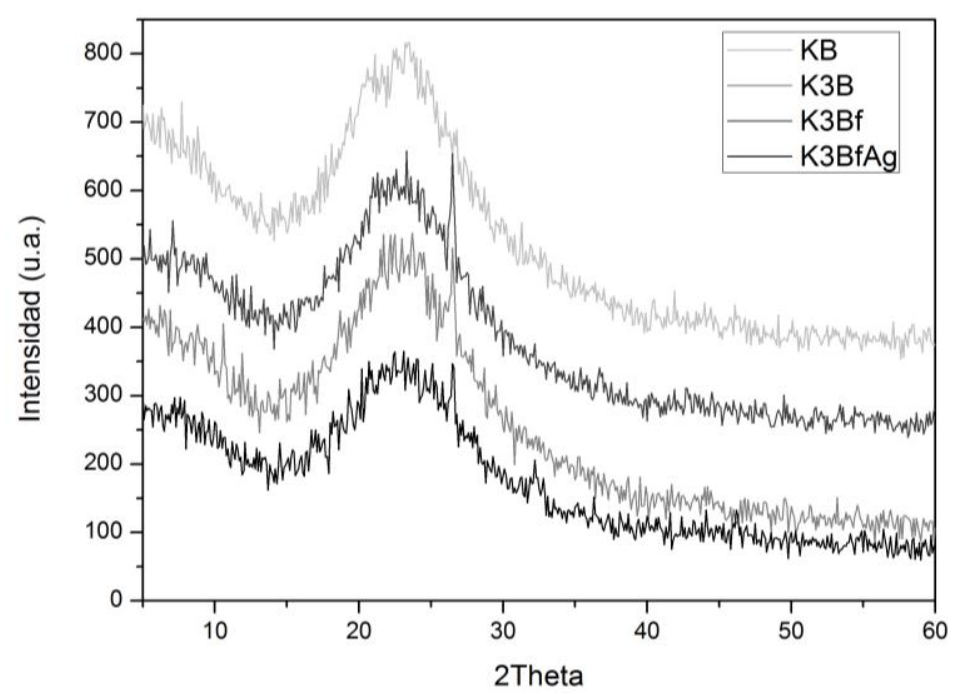

Figura 3: Diagramas de XRD de las muestras sintetizadas con TEOS y hidróxido de amonio como catalizador, sin carbón (KB), con 10,0\% de C (K3B), funcionalizada con APS (K3Bf) e impregnada con 1,0\% de nitrato de Ag (K3BfAg). 
En las micrografías SEM (Figura 4), se puede apreciar que las partículas presentan una morfología mayoritariamente redondeadas. Dichas partículas forman tipos racimos de tamaño variable, y esto puede deberse a la acción del hidróxido de amonio, lo que disminuye la estabilidad de la sílice obtenida con TEOS, generando una pronunciada disminución del área específica, como se visualiza en la Tabla 2.
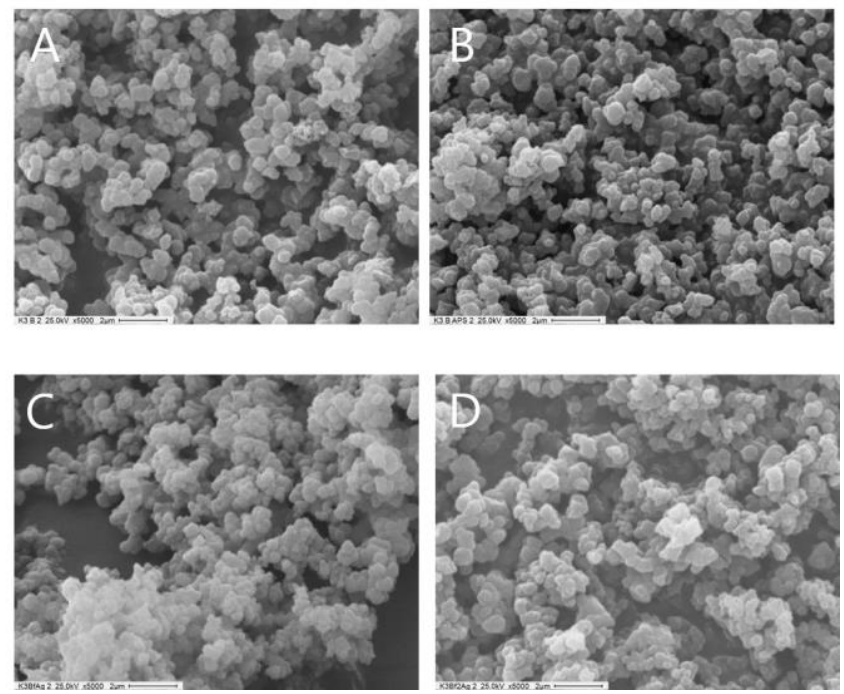

Figura 4: Micrografías SEM de las muestras (A) K3B, (B) K3Bf, (C) K3BfAg y (D) K3Bf2Ag. Magnificación 500x.

Finalmente, en la Figura 5 se muestran las micrografías TEM comparando las muestra KB y KB3, funcionalizadas y con diferentes porcentajes de $\mathrm{Ag}$ : KBf, KBfAg, KBf2Ag, K3Bf, K3BfAg y K3Bf2Ag., respectivamente. En base a las micrografías puede decirse que se visualiza la presencia de partículas de $\mathrm{Ag}$ dentro de la matriz de silice y encontrándose la mayoría en el orden de los $10 \mathrm{~nm}$, apreciando que la distribución de las partículas de plata es uniforme.
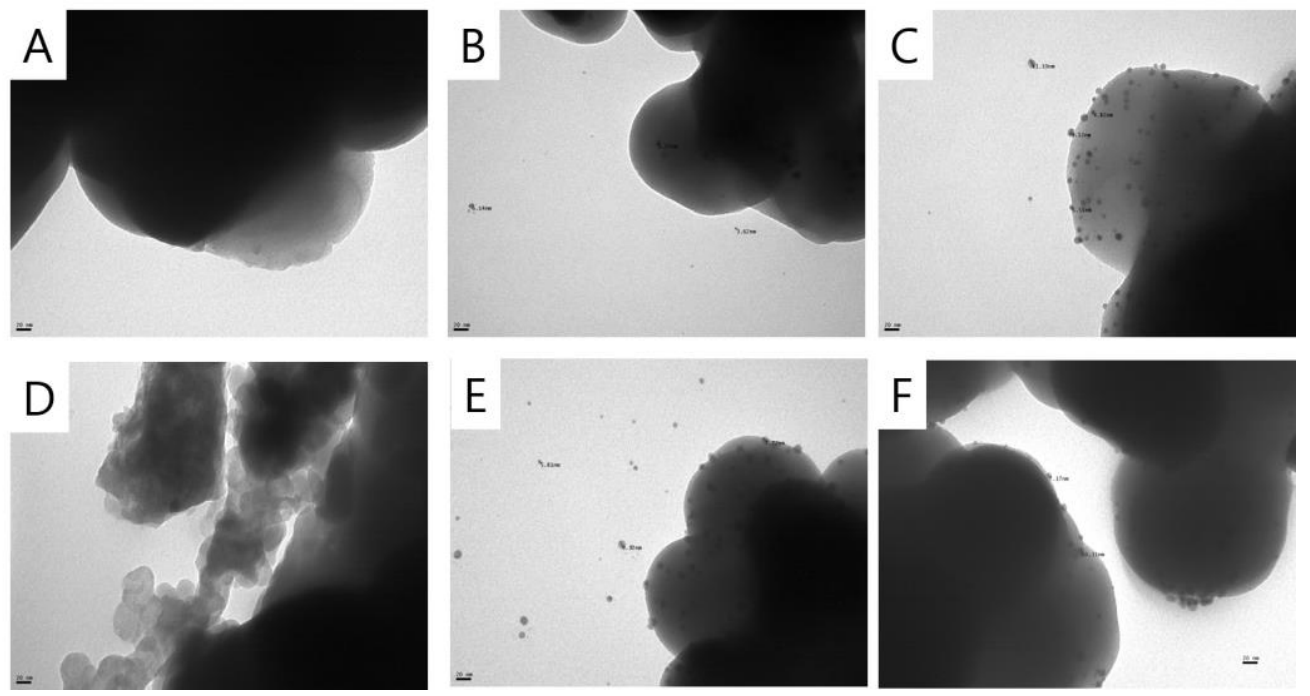

Figura 5: Micrografías TEM de las muestras (A) KBf, (B) KBfAg, (C) KBf2Ag, (D) K3Bf, (E) K3BfAg y (F) K3Bf2Ag. Magnificación 270000x 
A partir de las mediadas del diámetro de crecimiento radial de los cultivos efectuados en el ensayo de actividad antifúngica se calculó el porcentaje de inhibición (I) según la siguiente ecuación:

I \% $=[(\mathrm{C}-\mathrm{E}) / \mathrm{C}] \mathrm{x} 100$

Donde: $\mathrm{C}$ y E corresponden a los diámetros promedio de crecimiento radial de cada hongo en las placas de control y en las placas con los productos evaluados, respectivamente. Además, se determinó la desviación estándar entre medidas. En la Figura 6, se muestra los valores en un gráfico de barra tanto para las concentraciones 60 ppm y 120ppm de Ag en placa.
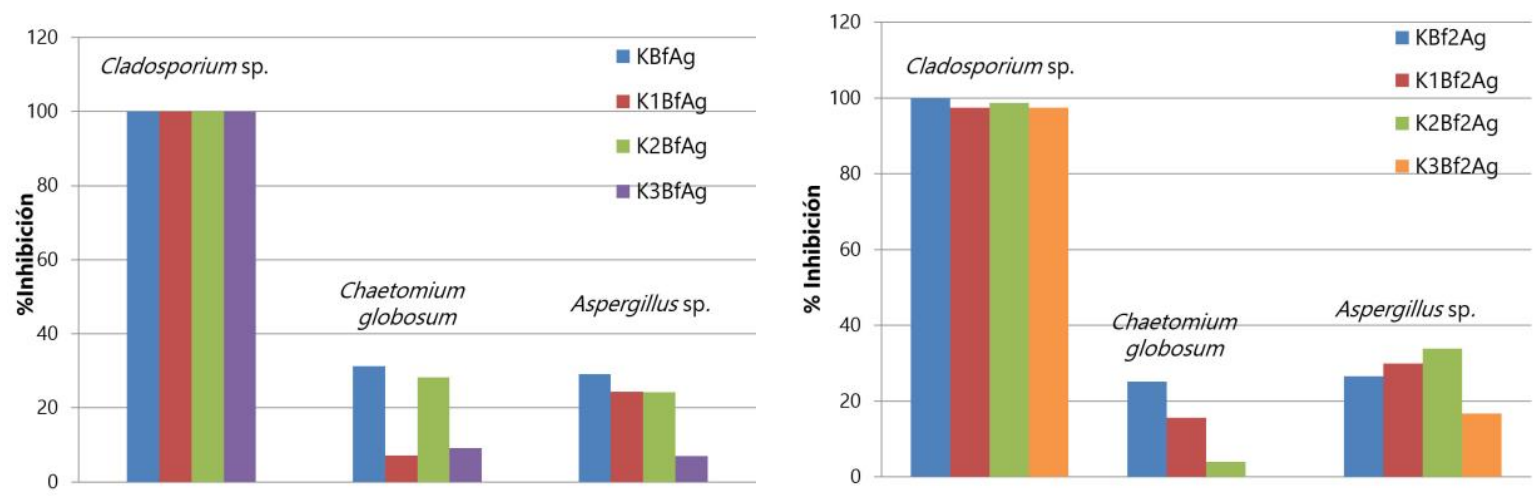

Figura 6: Ensayo de actividad antifúngica de los sólidos sintetizados.

Analizando los valores de \% inhibición obtenidos se puede observar que para los tres hongos no hubo cambios significativos en su crecimiento cuando el contenido de Ag en los sólidos era mayor, a su vez las muestras con cantidades de carbón intermedias presentaron una mayor efectividad. Asimismo, se pudo observar que el Cladosporium sp. es más sensibles a los sólidos ensayados en general y su crecimiento se vio totalmente inhibido en estas condiciones. Estos mismos solidos se ensayaron con hongos pertenecientes a distintos géneros, los cuales presentaron distintos comportamientos lo que puede observarse en la Figura 6.

En la Figura 7 se muestra como ejemplo las fotografías digitales del ensayo con los hongos Cladosporium sp. y Aspergillus sp., en los cuales se puede observar que tanto la muestra K1BfAg como la K1Bf2Ag inhibieron totalmente el crecimiento del primero, mientras que para el segundo se observó menor crecimiento y esporulación.

A
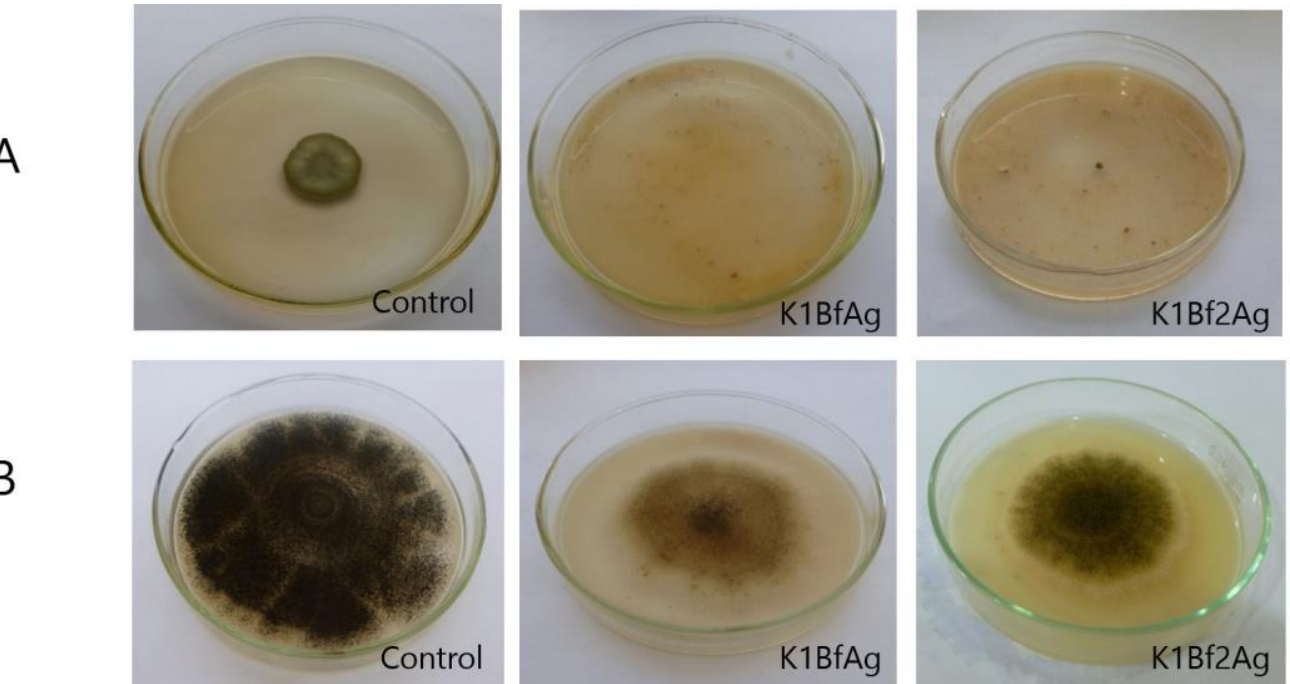

Figura 7: Fotografías del ensayo antifúngico de los sólidos sintetizados frente (A) Cladosporium sp. y (B) Aspergillus sp. 


\section{CONCLUSIONES}

Los resultados mostraron que las muestras que contenían menor concentración de carbón fueron las más efectivas en la inhibición del crecimiento de los tres hongos utilizados en el bioensayo. En una siguiente etapa se realizarán modificaciones en la síntesis del material silíceo para aumentar su efectividad.

\section{AGRADECIMIENTOS}

Los autores agradecen la colaboración a Mariela Theiller por las imágenes de SEM, a Lilian Osiglio por las titulaciones potenciométricas y a Pablo Fetsis por los estudios de propiedades texturales.

\section{BIBLIOGRAFÍA}

[1] ANASTAS, P.T. Y WARNER J.C., Green Chemistry: theory and practice, Oxford University Press, New York, U.S.A, Ed. S. Publications, 1998.

[2] IGAL, K., ARRECHE, R.A., SAMBETH, J.E., et al., "Síntesis y caracterización de matrices silíceas modificadas con carbón extraído del reciclado de pilas"; Anales CONAMET/ SAM, pp. 357-358, Nov. 2015.

[3] EL-RAFIE M.H., AHMED H.B., ZAHRAN M.K., "Characterization of nanosilver coated cotton fabrics and evaluation of its antibacterial efficacy”, Carbohydrate Polymers, v. 107, pp. 174-181, Jul. 2014.

[4] BELLOTTI N., ROMAGNOLI R., QUINTERO C., et al., "Nanoparticles as antifungal additives for indoor water borne paints", Progress in Organic Coatings, v. 86, pp. 33-40, 2015.

[5] JASIORSKI, M., LESZKIEWICZ, A., BRZEZIŃSKI, S., et al., "Textile with silver silica spheres: its antimicrobial activity against Escherichia coli and Staphylococcus", Journal of Sol-Gel Science and Technology, v. 51, pp 330-334, Sep. 2009.

[6] MARAMBIO-JONES C., HOEK E. M. V., "A review of the antibacterial effects of silver nanomaterials and potential implications for human health and the environment", Journal Nanoparticle Research, v. 12, pp. 1531-1551, 2010.

[7] SIMONCIC B., TOMSIC B., "Structures of Novel Antimicrobial Agents for Textiles - A Review", Textile Research Journal, v. 80, pp. 1721-1737, 2010.

[8] MAHLTIG B., FIEDLER D., FISCHER A., et al., "Antimicrobial coatings on textiles-modification of sol-gel layers with organic and inorganic biocides", Journal Sol-Gel Science and Technology, v. 55, pp. 269277, Sep. 2010.

[9] ROMANELli G. P., PASQUALE G., SATHICQ A. G., et al., "Synthesis of chalcones catalyzed by aminopropylated silica sol-gel in conditions of solvent-free"; Journal of Molecular Catalysis A: Chemical, v. 340, pp.24-32, 2011.

[10] ARRECHE, R. A, HERNÁNDEZ, F., BLANCO, M., et al., "Additive Synthesis by Sol-gel Method for its Use as Antimicrobial", Procedia Materials Science, v. 8, pp. 397-405, 2015. 